\title{
Analysis of Theta Power in Hippocampal EEG During Bar Pressing and Running Behavior in Rats During Distinct Behavioral Contexts
}

\author{
Bradley P. Wyble,${ }^{1 *}$ James M. Hyman, ${ }^{2}$ Christina A. Rossi, ${ }^{2}$ and Michael E. Hasselmo ${ }^{2}$
}

\begin{abstract}
These experiments examine changes in theta power as measured by wavelet analysis in five rats performing a conditional visual discrimination task and a simple running task. In the conditional task, rats were trained to press one lever to initiate a trial and then to press one of two choice levers, each corresponding to one of two cue lights. Analysis of theta power in this operant task found a large decrease in theta power during the choice bar presses, in contrast to the increase in theta power during trial initiation bar presses. This result seems to stand counter to results that propose consistent relationships between motor actions and theta power (Vanderwolf, EEG Clin Neurophys 26:407-418, 1969), as well as studies suggesting that the lack of bar-press theta is the result of habituation. However, these data can be seen as being in broad agreement with the theoretical framework of sensorimotor integration (Bland and Oddie, Behav Brain Res 127:119-136, 2001). To investigate further the power of theta observed at the termination of type 1 motor activity, a runway task was devised in which rats ran back and forth between two ends of a linear track, one of which was always rewarded and the other never rewarded. Theta power decreased sharply $240 \mathrm{~ms}$ before movement ended at the rewarded end, but not at the unrewarded end of the track. These data extend the current scope of theory in demonstrating that hippocampal theta activity can end abruptly 200-400 ms prior to the end of type 1 motor movement when approaching the end of a motor sequence. $\odot 2004$ Wiley-Liss, Inc.
\end{abstract}

KEY WORDS: wavelet; sensorimotor; learning; motor

\section{INTRODUCTION}

The theta rhythm is an oscillation in the range of $3-12 \mathrm{~Hz}$ that occurs within the limbic system of mammals during periods of alert immobility (Sainsbury et al., 1987a,b; Berry and Seager, 2001), voluntary movement (Vanderwolf, 1971; Sainsbury, 1970), and rapid eye movement (REM) sleep (Whishaw and Vanderwolf, 1973; Winson, 1974). It can also be found in humans, in the range of $4-8 \mathrm{~Hz}$ (Walter and Walter, 1953; Sarnthein et al., 1998; Kahana et al., 1999). Since its discovery, an enormous amount of empirical and theoretical work has attempted to discern the precise behavioral correlates and functional role of this oscillation.

In rat, the hippocampal theta rhythm occurs almost without exception during large-scale voluntary motor behaviors, such as ambulation, jumping, rearing, and bar pressing (Whishaw and Vanderwolf, 1973). Active investi-

\footnotetext{
${ }^{1}$ Computing Laboratory, University of Kent, Canterbury, Kent, United Kingdom; ${ }^{2}$ Psychology Department, Boston University, Boston, Massachusetts

Grant sponsor: National Institute of Mental Health (NIMH); Grant number: MH60013; Grant number: MH61492; Grant number: MH 60450.

*Correspondence to: Bradley P. Wyble, Computing Laboratory, University of Kent, Canterbury, Kent, UK CT2 7NF. E-mail: Wyble@mind.bu.edu Accepted for publication 26 January 2004

DOI 10.1002/hipo.20012

Published online 16 March 2004 in Wiley InterScience (www.interscience. wiley.com).
}

gatory behavior, especially sniffing, even without largescale movements, is also associated with theta (Green and Arduini, 1954; Vanderwolf, 1969). Automated behaviors, such as eating, drinking, grooming, and sexual behavior, are rarely associated with theta oscillations (Sainsbury, 1970; Vanderwolf, 1969). Furthermore, manipulations that evoke hippocampal theta (e.g., carbachol infusions in the medial septum) also tend to evoke the sort of behavior that normally accompanies theta (Monmaur and Breton, 1991; Lawson and Bland, 1993). Precise lesions of the medial septum (as opposed to the entire septal region) eliminate theta and also produce hyperplacid behavior (Clody and Carlton, 1969). Two types of hippocampal theta rhythmic activity have been observed, named type 1 and type 2 by Kramis et al. (1975). Type 1 theta is that which generally accompanies movement of the type described above; it is resistant to the effects of centrally administered atropine. Type 2 theta is more rarely observed in the rat, occurs during immobility under particularly stressful circumstances (Sainsbury et al., 1987a,b), and is sensitive to atropine.

The distinction between the theta correlates of voluntary and automated motor behaviors is not entirely clearcut, however. The act of pressing a weighted lever should lie clearly within the domain of voluntary motor activity, providing an expectation of an association with strong theta rhythmic activity. This is not the case, however. It is noted that bar-pressing theta tends to be weak (Whishaw and Vanderwolf, 1973) and in some cases disappears entirely with habituation (Feder and Ranck, 1973; Coleman and Lindsley, 1975). One of the aims of these experiments is to examine theta power accompanying bar presses in order to address this controversy. Discrepancies in results such as these could result from issues of relative timing of the bar press to other elements of the task.

Another element that is lacking from most data concerns the timing of the relationship between changes in behavior and changes in the theta rhythm. To date, analysis methods have depended largely on basic fast Fourier transforms (FFT), which provide good frequency resolution, but are capable of only limited temporal resolution (generally to a minimum of $500-800 \mathrm{~ms}$ for $7-\mathrm{Hz}$ signals). This article discusses an analysis of theta correlates of behavior based on wavelet transforms. Wavelet analysis provides a way of examining oscillatory power with temporal precision. In the case of this analysis, time windows of analysis are 60 and $200 \mathrm{~ms}$. 
Data from two experiments will be analyzed. The first addresses the presence of theta power during lever pressing behavior in a conditional discrimination task. It will be demonstrated that the profile of theta power within a trial changes as a result of training and subsequent partial reversal of that task. It will further be demonstrated from this same dataset that there is a very marked difference between the quantities and time courses of theta power associated with different types of lever pressing behavior in this task. The second experiment involves a single track runway and compares theta power measured as a rat is running toward a rewarded area versus theta power as a rat is running toward a nonrewarded area. It is hypothesized that quantifiable differences in theta power will be found between similar motor activities embedded in different behavioral contexts within both of these experiments.

Both experiments demonstrate very rapid reductions in theta power that occur during particular motor activity at the end of a sequence of activity necessary to obtain food reward. The results will be discussed in relation to theories of the functional correlates of hippocampal theta activity, focusing on the sensorimotor integration hypothesis of Bland and Oddie (2001). This theoretical analysis of theta generation mechanisms and correlates posits that hippocampal theta activity plays an active role in the mediation of motor activity. In this framework, type 1 theta (noncholinergic) directly supports the initiation of motor activity and also the integration of sensory information into motor programs. Type 2 theta (cholinergic) is considered a priming signal to prepare the motor system for activation, but it can also provide quantitative information to modulate the intensity of an ongoing motor program. At the end of the discussion, an alternative theory will be presented that posits two distinct forms of attention to sensory cues, mediated by theta and non-theta mechanisms. This theory is broadly compatible with the sensorimotor hypothesis, but it is more specific about the interaction of theta activity and incoming sensory information.

\section{MATERIALS AND METHODS}

\section{Surgery and Electrodes}

Male Long Evans rats were implanted with vertically distributed pairs of electrodes in the dorsal hippocampus aimed at crossing the theta phase reversal point (Winson, 1974) such that the deep electrode would record theta near the hippocampal fissure. The other electrode tip would record theta from a point above the phase reversal zone near stratum radiatum. The result was theta of opposite phase recorded from the two electrodes when referenced to a ground screw near the cerebellum. All data in the present report refer to theta recorded from the deeper electrode, near the hippocampal fissure (commonly known as fissure theta). Figure 1 provides an example of theta obtained from two properly positioned electrodes. The larger-amplitude signal was always recorded from the deeper electrode.

Rats were anesthetized with halothane as provided by a gas anesthesia rig (Harvard Apparatus), while electrodes were lowered into position at $-3.5 \mathrm{~mm}$ anteroposteriorly (AP) and $2 \mathrm{~mm}$ later-

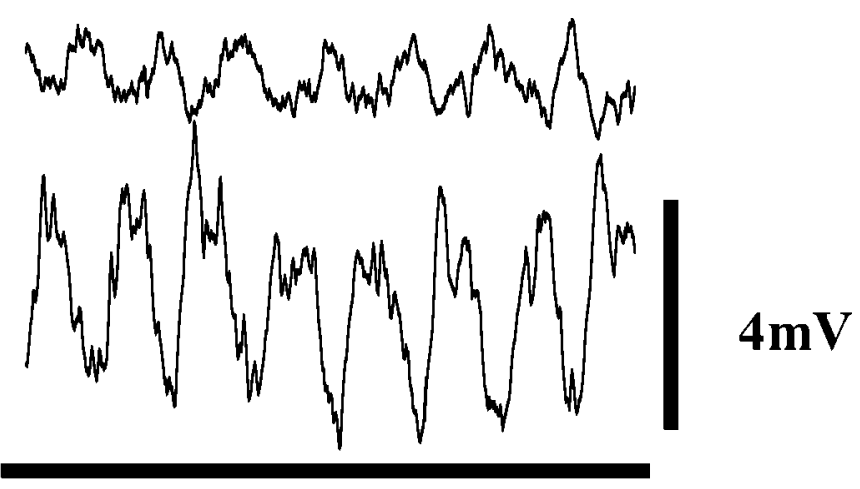

\section{Second, $7.7 \mathrm{hz}$}

FIGURE 1. Theta obtained simultaneously from two electrodes straddling the phase reversal point of hippocampal theta. The lower trace is from the deeper electrode, near the hippocampal fissure.

ally. Two of the rats used in this study were implanted with stationary $100-\mu \mathrm{m}$ electrode bundles positioned manually during surgery. Other rats were implanted with $30-\mu \mathrm{m}$ electrode bundles, using adjustable drivers. These electrodes were lowered into position to find optimal theta recording depth following recovery. The two rats with $100-\mu \mathrm{m}$ stationary theta recording electrodes also had 30- $\mu \mathrm{m}$ electrode bundles implanted in the medial septum ( 0.7 AP, $0 \mathrm{~mm}$ laterally) to collect spiking data not analyzed in this report.

Recording electrodes were fashioned from stainless steel wires coated in Formvar. Encephalographic (EEG) electrodes in the hippocampus were 30 or $100 \mu \mathrm{m}$ in diameter, cut with a diagonal angle. The recorded signals were pre-amplified through a J-FET headstage made by NB-Labs (http://www.nblabslarry.com) to reduce motion artifacts and were then amplified with a Neuralynx amplifier before being passed to a Data Translation A/D board for storage by the DataWave Discovery package. EEG signals were recorded at $510 \mathrm{~Hz}$ and were amplified 500 -fold with a $0.1-\mathrm{Hz}$ to $325-\mathrm{Hz}$ bandpass hardware filter within the Neuralynx amplifier.

\section{Histology}

Histology was performed in a similar manner as in previous research (Wyble et al., 2000). Four of the initial animals in this research underwent full histology with a Prussian Blue reaction on neutral red staining to confirm that the electrodes were located at the hippocampal fissure despite minor variation in stereotaxic coordinates. For all animals in this experiment, obtaining phase reversed theta recordings at appropriate depths was the primary means for determining the accuracy of electrode placement. A demonstration of phase-reversed theta signals recorded concurrently from two electrodes in one rat is given in Figure 1. No rats for which simultaneous, phased reversed theta signals could not be obtained were used in this experiment. Data from the deeper electrode, corresponding to dentate fissure theta, were always used for analysis. These fissure theta oscillations were always substantially 
larger in amplitude than the signal from the electrode near to CA1, as can be seen in Figure 1.

\section{Wavelet Analysis}

Wavelet analysis was used with the morlet basis function provided within the MATLAB wavelet toolbox to determine the power of theta oscillatory activity. Wavelet analysis allows greater temporal resolution than do FFTs. An FFT is generally used to provide a measurement of power at different frequencies within a given segment of a signal, but it works best with a lengthy signal (approximately $1 \mathrm{~s}$ would work well with these data). Wavelet analysis allows the power at a given range of frequencies to be analyzed accurately at a resolution closer to the sampling frequency of the signal. In these data, wavelet data on theta power was taken at 60- and 200-ms intervals.

The morlet wavelet is chosen as a basis function because of its sinusoidal shape and declining envelope. A precedent for using the morlet as an index of theta activity is found in Caplan et al. (2001). The morlet function, being symmetrical, provides output with a phasic component that was eliminated by averaging together the absolute value of signals over 60 - and 200 -ms intervals. The bands of the wavelet analysis output that were designated as indicative of theta power were chosen by visual inspection of concurrent EEG and wavelet output, and corroborated by passing artificially generated sine waves of known frequency into the wavelet analysis. For the low-resolution wavelet analysis, theta power comprised the frequencies $6.5-10.5 \mathrm{~Hz}$. For the high-resolution analysis, the theta band encompassed the frequency range $4-9.5 \mathrm{~Hz}$. Theta power recorded in this experiment was generally constrained to the range $7.5-8.5 \mathrm{~Hz}$. In all cases for which reductions of theta power were recorded, visual inspection of the wavelet averages confirmed that these reductions were not caused by a drift of the dominant theta frequency to a range approaching or crossing the edges of the defined theta bands.

This method of frequency analysis includes some temporal contamination, in that an abrupt signal change can be observed to affect the wavelet analysis results for some period prior to the actual event. Artificially generated $8.5-\mathrm{Hz}$ transients using the data analysis routines described here demonstrate that a $12 \%$ signal change can be observed $90 \mathrm{~ms}$ both before and after the actual event. This artifact places some constraints on the temporal precision of the analysis performed here, but such contamination is largely confined ( $75 \%$ of the total signal change) to a 180 -ms window, symmetrically centered at the time of the transient ( $90 \mathrm{~ms}$ prior to and $90 \mathrm{~ms}$ following). Practically all such contamination (96\%) is contained within a $300-\mathrm{ms}$ window $(150 \mathrm{~ms}$ prior to and $150 \mathrm{~ms}$ following the event). Note that this artifactual effect is too short to explain the reductions in theta power observed prior to cessation of motor activity described below in the results.

\section{Normalization}

Theta power, as measured by wavelet analyses, varies widely between rats due to differences in electrode placement and electrode construction. There is also a general trend for theta power to decrease over time due to habituation to the task and the environ-
A.

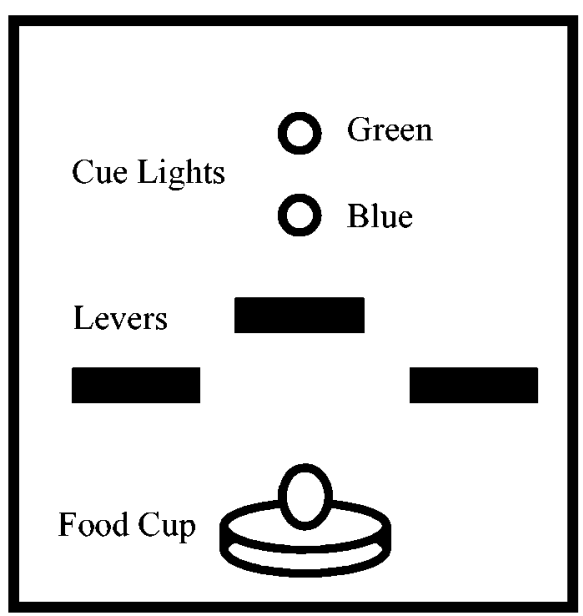

B.

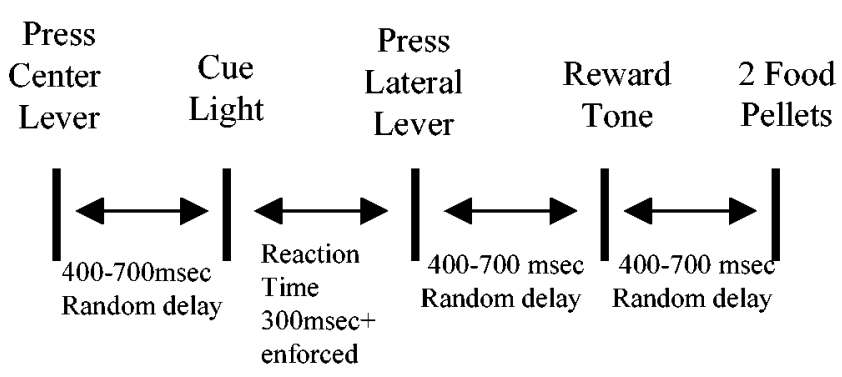

FIGURE 2. A: Front of the panel used in the operant chamber for the conditional visual discrimination task. B: Temporal structure of a correct trial. An incorrect trial would lack the reward tone and food pellet elements.

ment. The phenomenon of a habituation induced drop in theta power has been previously established (Whishaw and Vanderwolf, 1973). Type 2 theta is also known to habituate in response to repetition of a sensory stimulus (Irmis et al., 1970).

To accommodate this reduction in theta power, this analysis focuses on theta power changes relative to a chosen point in time, $1400 \mathrm{~ms}$ prior to the event in question. In order to compare theta between rats and between different behavioral epochs (these epochs are described in detail below) within the data of a given rat, theta power data were normalized twice. First, for each subset of data corresponding to one epoch for a rat, theta power at each point in time was divided by the average theta power of that epoch to bring the mean to 1.0. These data were then averaged over the epoch to form a single theta power profile for one rat across one epoch. These curves were averaged across rats in an unweighted fashion; the resulting curve was normalized by dividing all values by the mean theta power of the first data point in the analysis, 1400 ms prior to an event. A similar process was performed for the running track theta, with theta power being normalized per food/no food condition within each rat, averaged across rats and then normalized again to the first time point in the analysis, 1060 $\mathrm{ms}$ prior to the end of running. 


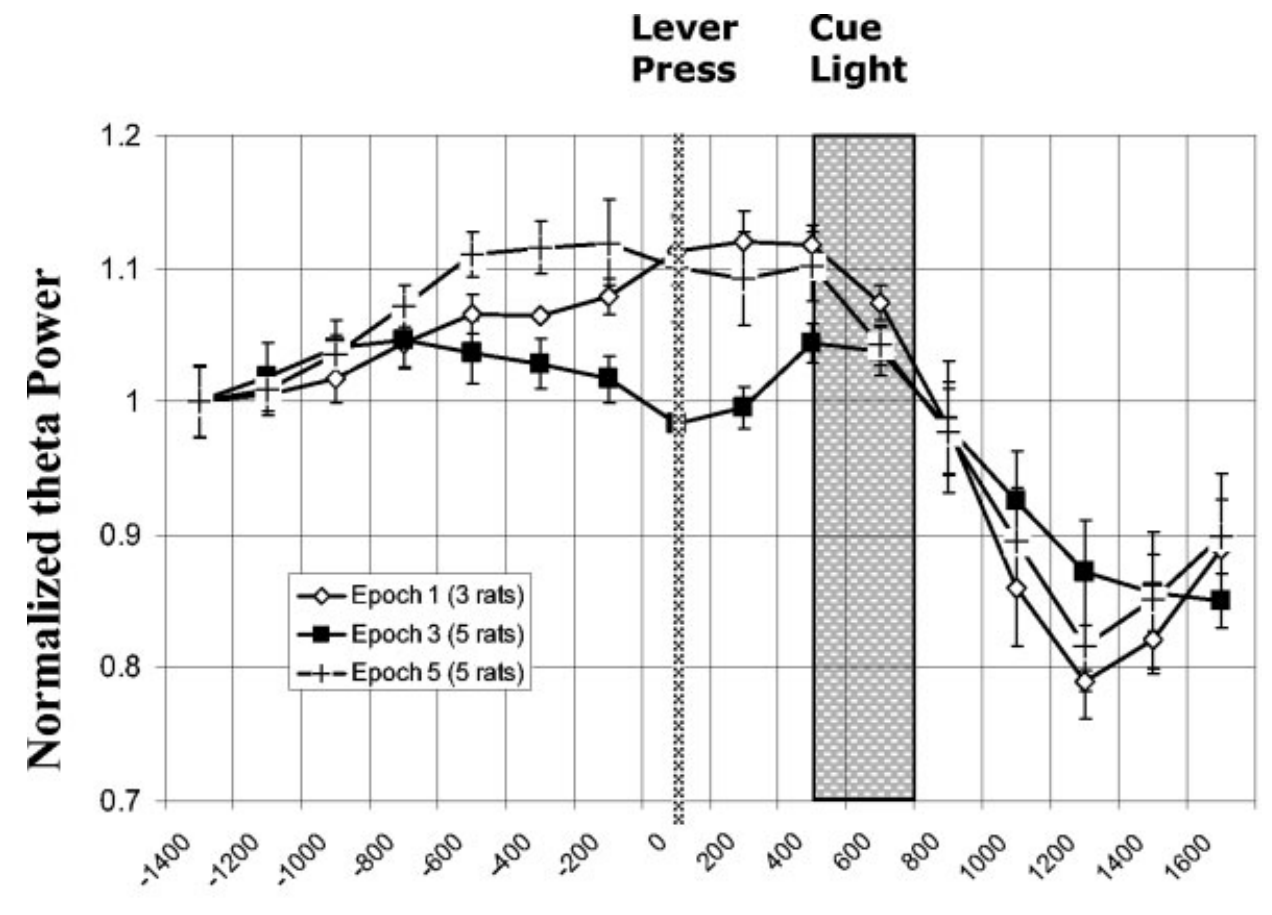

\section{Time relative to Lever Press event (msec)}

FIGURE 3. Data showing theta power averaged time locked to center lever presses in three epochs: epoch 1 (pre-acquisition, chance performance: open diamonds), epoch 3 (acquired, $75 \%$ performance: filled squares), and epoch 5 (post-reversal, chance performance:

\section{Experimental Protocols}

\section{Conditional visual discrimination task}

Behavioral apparatus. The behavioral chamber is composed of sheets of flexible black 1/8-inch polyvinyl chloride (PVC) arranged in a box 24 inches long, 14 inches wide, and 15 inches high. A PVC panel inside the box (Fig. 2), which contains the levers, lights, and food well, sits 8 inches from the wall, leaving a 16-inch by 14-inch chamber for the rat. The top of the box is open for visual monitoring and free motion of the recording cable. A water nozzle extends into the box through the wall opposite this panel.

The intelligence panel contains three levers, two of which are 6 inches apart and 4 inches above the floor, while the third is centered at a height of 6 inches. A 12 Volts Direct Current (VDC) blue and a 24 VDC green light of different design and brightness are located 2 and 7 inches above the center lever, respectively. The food well is a plastic cup lying beneath the center lever. A food dispenser behind the panel delivers single 45-mg pellets to the food well via a small (1-cm-diameter) hole. All the levers and lights and the food dispenser are computer controlled via digital interface.

Task structure. Figure 2 also illustrates the components of the operant task used in these experiments. The operant task required rats to press the single lever once to initiate a trial at any time following illumination of the house lights. After a randomized delay of $400-700 \mathrm{~ms}$ following this center lever press, one of the crosses). Error bars are for unweighted averages with one data point per time interval per rat. In each trial, time is aligned to the lever press (vertical line). The time of the cue light is variable relative to the center lever, indicated by the gray shaded region.

two cue lights illuminated, signaling which of the two lateral levers was active for that trial. Rats could then press the correct lateral lever to complete a trial correctly. Incorrect responses ended the trial immediately with no possibility of correction.

On a rewarded trial, a tone sounded for $500 \mathrm{~ms}$ after the lateral lever press. For three of the rats used in this study, there was an additional delay of $400-700 \mathrm{~ms}$ between the lateral lever press and the reward tone. Two food pellets (Noyes Pellets, Formula P) were delivered in immediate succession after a randomized delay of $400-700 \mathrm{~ms}$ following the start of the reward tone. The delivery of each food pellet was preceded by a loud clicking sound from the electrical dispenser.

Experimental protocol. Following a 2-week recovery period, electrode depths were adjusted to obtain optimal theta for some of the rats, depending on the type of implant. Rats were shaped to perform the task, first by receiving food reward for pressing lateral levers and later by incorporating the center lever into the task. During the shaping period, the cue lights were never active. Recorded experiments always began with the first exposure to the cue lights, which corresponded to the first trial of epoch 1.

In all animals, EEG and unit data were recorded continuously during experimentation. Event codes noted the time of cue lights, lever presses, tones, and food deliveries with time stamps that were synchronized with the time stamps of EEG records to ensure accurate timing of data. 
A daily experiment consisted of an approximately 30-min exposure to the operant chamber. Because of periodic difficulties involving premature release of the headstage from its holding pins, some trial sets were shorter than this.

Rats were trained on the task until they were able to perform 20 correct trials in a row. At this point the contingency was reversed. Contingency reversals involved switching the association between the two lights and the two levers after rats were able to perform 20 consecutive trials correctly. For example, the reversal could make the task contingency shift from the green/top light indicating that the left lever was rewarded, to the green/top light indicating that the right lever was rewarded. Rats were trained extensively on the reverse contingency although none were able to relearn the task after reversal in a suitable amount of time.

Data analysis. Data were selected from rats for each trial with both one center and one lateral lever press in the proper order, regardless of the accuracy of the left or right response. This analysis would exclude trials in which the rat failed to push either lateral lever for the length of the trial, or in which the rat depressed a lateral lever to initiate a trial. A successful trial therefore consists of at least three elements: depression of the center lever, illumination of the cue light, and finally depression of a lateral lever. A successful correct trial also contains a food tone and a food drop. For each successful trial, theta power was determined for eight 200-ms bins before and after the center lever press.

Behavioral trials were combined across days to construct a single combined dataset across which the rat's performance in the discrimination task could be tracked, and used as an index for delineating sets of trials corresponding to similar behavioral epochs between rats. This combined dataset was separated into five epochs for each rat, corresponding to pre-acquisition, acquisition, asymptotic performance (acquired), reversal, and post-reversal phases of learning:

Epoch 1: The pre-acquisition epoch (epoch 1) included trials for which performance was at or near $50 \%$.

Epoch 2: The acquisition epoch (epoch 2) included trials as performance increased to asymptote.

Epoch 3: The acquired epoch (epoch 3) included all trials at asymptotic performance levels (greater than $75 \%$ for all rats).

Epoch 4: The reversal epoch (epoch 4) included trials from the trials just after the reversal of the behavioral contingency until performance had risen back to the $50 \%$ point.

Epoch 5: The post-reversal epoch (epoch 5) included all trials at or near the $50 \%$ performance level after the reversal.

Data from epochs 2 and 4 (the transition epochs) strongly resembled an average of results from epochs 1 and 3 and 3 and 5. For this reason, and also to provide the simplicity of analyzing data collected during static, as opposed to dynamic, behavior patterns, the epoch-based analysis in the present study focuses entirely on epochs 1, 3, and 5-periods during which behavior was stable at accuracy rates of $50 \%, 75 \%$, and $50 \%$, respectively.

For two of the five rats used in this experiment, data collected during epoch 1 and part of epoch 2 were invalid due to a timing error in the code controlling the task, which frequently made the center lever press unnecessary to activate a cue light and obtain reward. The data from these rats are only considered for epochs 3 through 5 even though valid trials could be found for epoch 1 .

Data were then normalized and averaged together across rats within epochs 1, 3, and 5, as described above. For each pair of epochs, a focused analysis of variance (ANOVA) was used to compare the theta power profiles (Microsoft Excel Statistics package: ANOVA with Replication; 16 time points, 2 conditions, three or five rats).

For the analysis of center and lateral lever theta, theta power profiles were constructed for lateral lever presses as described above for center lever presses. Data were collapsed across all 5 epochs for the three rats with completely valid data; across epochs 3, 4, and 5 for the two rats with partially valid data; and included epoch 1 data for a sixth rat that failed to learn the task entirely. The same process was performed for center lever data, and the theta power profiles were compared with a similar ANOVA as described above.

\section{Running task}

Behavioral apparatus. Rats ran along an elevated track 54 inches in length, 4 inches in width, with 1 -inch walls. The track and walls were composed of black PVC tacked onto a wooden base. Position data from a single spot tracking system, purchased from Datawave Technologies was recorded at approximately $60 \mathrm{~Hz}$. An LED cluster at the rear of the headstage provided the fixation for the position tracker.

Task structure. During initial training only, reward was given manually with two food pellets (Noyes, Sweetened Purified Diet) placed at each end of the track as the rat shuttled back and forth between the ends.

After a smooth behavioral repertoire of running from one end of the track to the other was established, reward was discontinued on one end of the track. Additional training was required to train the rats to travel to the unrewarded end of the track. Rewards were withheld for slow or intermittent movements until rats usually ran from one end to the other in an uninterrupted running motion. In like manner, rats were trained to remain at the unrewarded end for several seconds before returning by withholding food reward for runs in which the rat failed to pause at that end for $\geq 3 \mathrm{~s}$. This prevented rats from reaching the unrewarded end and returning immediately.

Data were then collected for at least five sessions on the one-food version of the task. Each session contained at least 20 runs, but varied in length dependent on the motivation of the animals. The rewarded end remained constant for all sessions, for all rats. Trips were then separated into two groups on the criteria of whether the rat was arriving at the rewarded or unrewarded end of the track based on head tracking data.

Data analysis. All data analysis was done using MATLAB 6.1 software from Mathworks. Position data were analyzed, and runs from one end of the track to the other were identified and marked. The identification of such a trip involved an algorithmic process 
that examined the rats' speed for certain patterns characteristic of large-scale locomotion from one end of the track to the other. Speed exceeding a threshold characteristic of a smooth trajectory at a running speed triggered a closer scan for the initiation and termination of the trip. Trips with ambiguous or uncertain initiation and terminal segments were discarded, as were those for which the average speed indicated a slower walking pace. Valid trips were segregated as rewarded or unrewarded by the location of their final segment.

For each valid trip, theta power was extracted as described above in 60-ms bins over a time interval of approximately $1 \mathrm{~s}$ prior to and following the end point of each trip. The end of a trip was defined to be the point at which speed reached an asymptotic low point. Each rat's theta power profiles for the selection of food and nonfood trips were averaged together and normalized by division by the first data point of the series as described above. These theta power profiles were averaged across rats for the food and no food conditions and an ANOVA was used to compare these theta power profiles (Microsoft Excel Statistics package: ANOVA with Replication; 34 time points, two conditions, five rats). A two-tailed $t$-test also compared individual time points to look for the first time point prior to the stopping at which theta power data were significantly different.

\section{RESULTS}

The results presented describe differences in theta power found within subjects. First, theta in response to center lever bar pressing was found to vary with changes in learned behavior on the conditional task. Theta power increased prior to the center lever press only for chance level (50\%) performance (that found in epochs 1 and 5). Second, theta power was found to vary between center (trial initiation) and lateral lever (choice) presses. A sharp drop in theta power was found prior to and during lateral lever presses. Finally, in the running task, theta power was found to drop sharply as the rats were decelerating toward a rewarded location. Deceleration toward the nonrewarded end did not produce such theta suppression.

\section{Theta Power Time Locked to Center Lever}

The theta power profile for all rats was remarkably consistent at the time of the center lever press. The pattern of theta power obtained is presented in Figure 3. The important point to take from this figure is that the theta profiles for epochs 1 and 5 are similar, while that of epoch 3 varies significantly. During chance level behavior (50\% accuracy, epochs 1 and 5), theta power increased before and during the center bar press event, and began to drop off immediately prior to the cue light illumination event. Theta power increased by a maximum of about $12 \%$ between the first time point of analysis $(-1,400 \mathrm{~ms})$ and the moment of the center lever press when averaged across the three rats which had valid data for epoch 1 . Theta also increased by $12 \%$ in epoch 5 for the combined data of all five rats. Figure 3 uses a low-resolution theta power profile, in which each time point represents $200 \mathrm{~ms}$ and the length of the entire trace is $3.2 \mathrm{~s}$. Error bars and ANOVAs are calculated based on one data point per rat per time point, which is a conservative measure given that each rat's data in a given epoch represent the average of 50-2,000 individual data points.

Figure 3 also shows that after the rats had been trained to discriminate between the lights to perform a correct bar press response (epoch 3), theta power did not show the large increase prior to the center lever press found in epoch 1 . Instead, theta power decreased by roughly $5 \%$ at the time of the center lever press, although this change only approaches significance, i.e., T(4), $P<0.12$, twotailed, when compared with the data point $200 \mathrm{~ms}$ prior. Theta power then increased, reaching a peak $400 \mathrm{~ms}$ after the center lever press, a time corresponding to the shortest possible randomized delay for the presentation of the cue light. Rats trained to discriminate between the lights generally assumed an immobile stance, resting on the center lever and staring at the lights during the delay period before choosing a lateral lever.

Reversing the light/lever contingency rapidly returned the animals to chance level performance. These post-reversal chance performance trials comprise epoch 5. As described above, theta power data from this epoch closely resembled that of epoch 1 , with a large increase in theta power prior to and extending through the center lever press behavior. A two-way ANOVA with repetition for the three rat data set shows that the theta power profile for epoch 3 is significantly different from epochs $1(\mathrm{~F}(1,64)=15.05, P<$ $0.001)$, and epoch $5(\mathrm{~F}(1,64)=14.34, P<0.001)$. Epochs 1 and 5 were not significantly different from each other $(\mathrm{F}(1,64)=0.13$, NS). An omnibus ANOVA comparing epochs 1, 3, and 5 for the three rats also revealed a significant difference $(\mathrm{F}(1,96)=7.72$, $P<0.001)$. The comparison of epochs 3 and 5 when all five rats were included (the data shown in Fig. 3) also revealed a significant difference $(\mathrm{F}(1,128)=5.59, P<0.02)$. No interactions were significant in any of these ANOVAs.

\section{Comparison of Center and Lateral Lever Presses}

Theta power recorded during and time-locked to center lever and lateral lever press were compared when collapsed across all behavioral epochs as shown in Figures 4 and 5. A 2-way ANOVA with repetition found a significant difference $(\mathrm{F}(1,160)=145.79$, $P<0.00001)$ between the theta power profiles of center and lateral lever presses across all behavioral epochs (Fig. 5). The interaction term was also highly significant $(\mathrm{F}(15,160)=8.22, P<0.00001)$. Center lever pressing behavior was accompanied by increasing theta power prior to the lever press behavior. Theta power tended to reach an asymptotic level $400 \mathrm{~ms}$ before the center bar press itself and maintained this level until $400 \mathrm{~ms}$ after the center lever press. This level of theta power was generally the largest in amplitude for any of the behaviors within a trial, especially during epochs 1 and 5 .

In contrast, theta power decreased sharply prior to pressing of the lateral levers. Theta power began to diminish $400 \mathrm{~ms}$ prior to the lateral lever press, reaching a maximum diminution $400 \mathrm{~ms}$ after the lever press. The magnitude of the reduction was substantially larger than the positive changes described in the previous section for the center lever, declining by $25 \%$ on average. Visual examination of multiple individual EEG records at time periods 
A. Single Trial EEG for Center Lever Presses

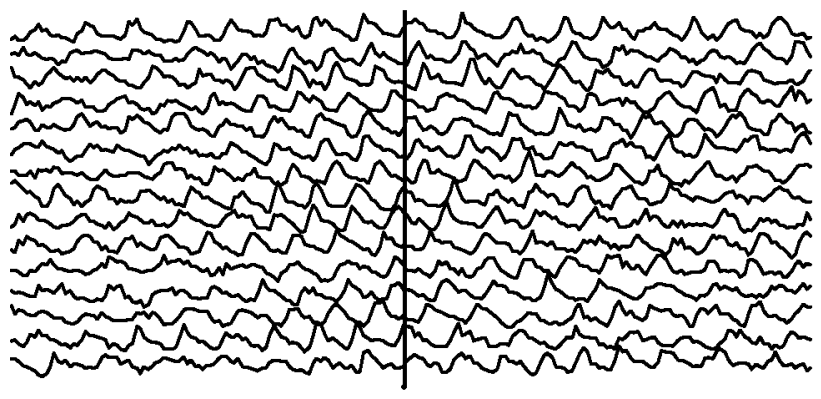

B.

Single Trial EEG for Lateral Lever Presses

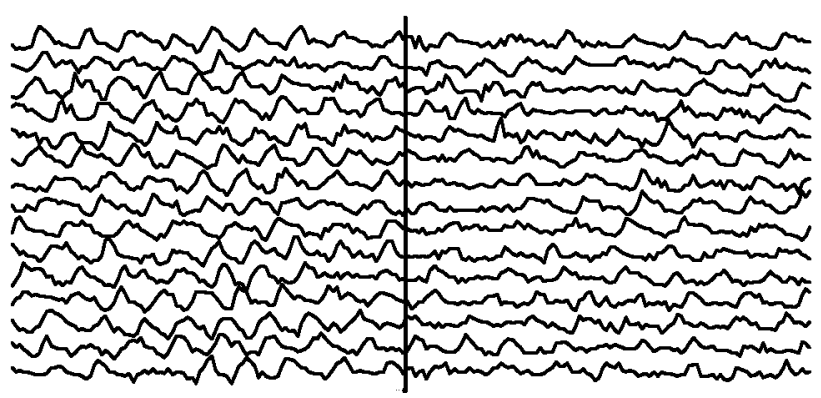

FIGURE 4. Single-trial EEG data. A: Fifteen consecutive center lever presses. B: Fifteen consecutive lateral lever presses. Samples are $2 \mathrm{~s}$ in length and centered at the time of the lever press. Note the strong theta oscillations observable throughout the center lever presses, but the abrupt decrease in theta amplitude associated with the lateral press.

associated with these lateral lever presses indicate that theta disappears almost completely in a large number of the records (Fig. 4). These two representative EEG datasets are each from sets of 15 consecutive trials within the same rat within epoch 1 .

EEGs recorded during center lever presses are markedly different, with no visible decrease in theta at the time of the center lever press behavior, especially during epochs 1 and 5, when center lever associated theta was strongest.

This marked difference in theta power profile between lateral and center lever presses was obtained throughout all epochs. The differences in theta power observed between epochs for center lever presses, as shown in Figure 3, were always dwarfed by the large drop in theta power associated with lateral lever presses at every epoch.

\section{Specific Differences in Motor Activity}

The motor behavior for depression of the lateral lever was somewhat different from that for depression of the center lever. Lateral lever behavior consisted of either a rapid movement from the center lever down to a lateral lever, or a return to a crawling position, a walking movement, and rearing to press the bar. The center lever depression invariably involved a rearing motion from a horizontal orientation. It is possible that the difference in theta power was a result of this difference in behavior. In addition, the lateral levers were somewhat closer to the ground than the center lever which meant that a slightly different posture was necessary to press it. Two separate analyses, described below, discount the possibility that specific motor differences were responsible for the differences in theta power accompanying center and lateral lever presses. These analyses find no difference between theta associated with lateral lever presses that had distinct behavioral repertoires as determined by the timing of behavioral data.

In many trials, rats erroneously reared and pressed a lateral lever instead of the center lever at the beginning of a trial. If the difference between the theta power profiles of center and lateral levers is a result of the physical differences between the motor sequences used in pressing the two levers, then the theta power of invalid lateral lever presses, which resembled center lever presses behaviorally, should differ from that of the lateral lever presses that occurred in the context of a valid trial. The theta power associated with these invalid lateral lever presses was not significantly different from that associated with the valid lateral lever presses $(\mathrm{F}(1,80)=2.39$, N.S.) for the time period up to and including $400 \mathrm{~ms}$ following the bar press.

A second difference in motor behavior for these lateral lever presses involved a side bias that all rats in this study developed, especially during well learned behavior (epoch 3). A rat would often rear and depress the center lever from a position midway between the center lever and its favored lateral lever. This lever bias could always be determined afterward by examining reaction times with consistently shorter reaction times indicating the favored lever. There was no detectable difference between the theta power associated with the favored and unfavored lateral lever press behaviors despite the differences in movement required to shift to the unfavored lever $(\mathrm{F}(1,128)=3.20, P>\mathrm{NS})$.

The lack of a difference in the theta power profiles between valid and invalid lateral lever presses as well as between favored and unfavored lateral lever presses indicates that differences in the actual motor activity associated with center and lateral lever pressing are not associated with differences in theta power that accompany those lever presses. This finding suggests that the differences in theta power were associated with expectation of reward or the end of a trial that accompanied the lateral lever press.

\section{Running Task}

Rats generally exhibited running behavior from one end of the track to the other, accelerating and decelerating quickly in a monotonic trajectory. At the rewarded end of the track, rats would sniff in the area the food pellets were placed in and consume them rapidly. The food pellets were small enough that consumption was rapid and barely interrupted the exploratory sniffing behavior of the rat. Note that speed measurements, as determined from head tracking data, never reached an absolute zero, as even sniffing or headshaking will register as a small baseline level of recorded movement.

Running to the unrewarded end of the track was slower than that to the rewarded end by $15-25 \%$ when measured by peak 


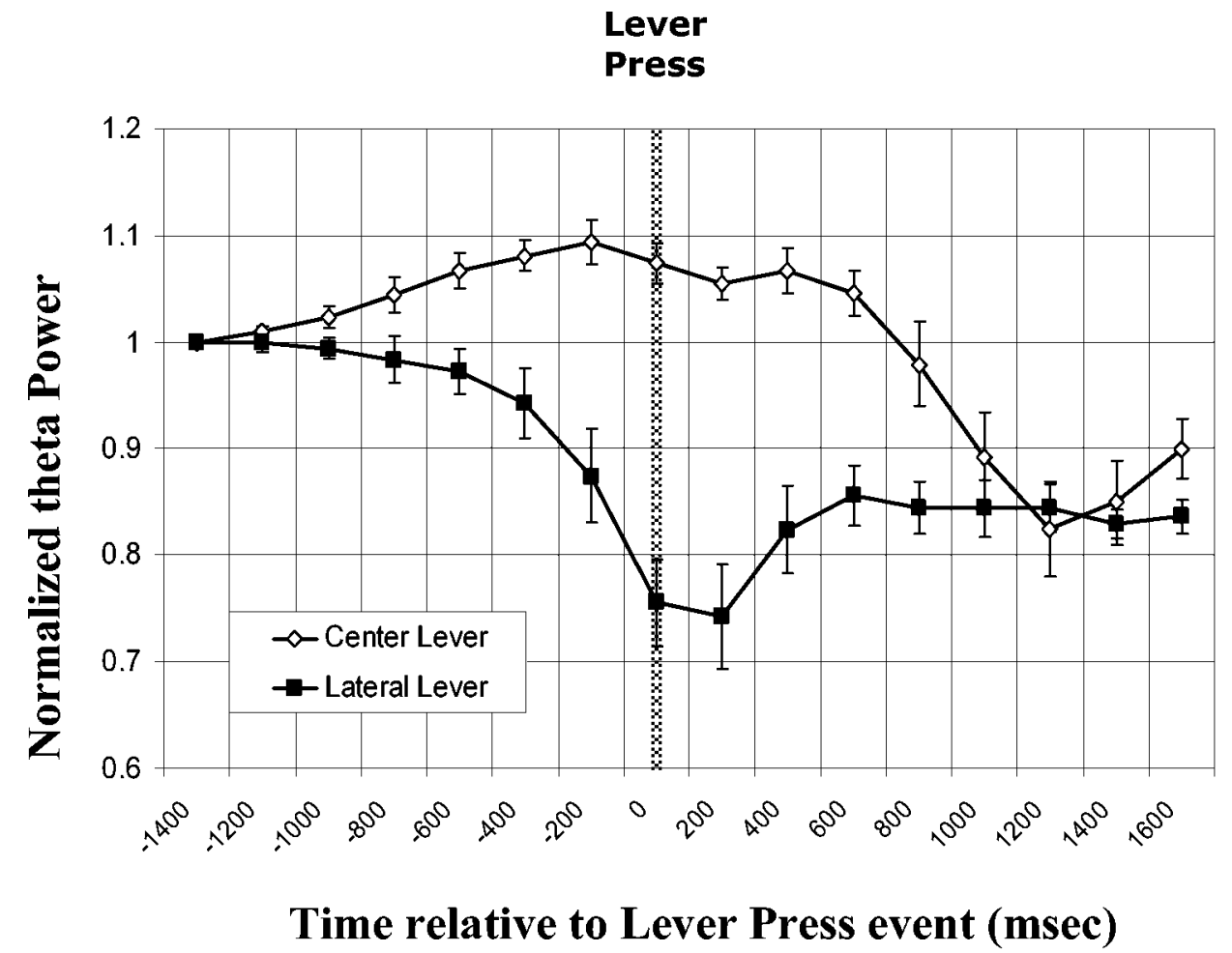

FIGURE 5. Theta power profiles averaged across rats and time locked to center lever (open diamonds) and lateral lever (filled squares) presses are displayed. Theta power increases prior to center lever presses, but decreases prior to lateral lever presses. The align-

velocity attained during the run. Rats running to the unrewarded end sometimes used a halting, walking gait. The trip detection algorithm rejected such behaviors from the analysis. Rat behavior at the unrewarded end of the track involved sniffing investigatory behavior and occasional rearing. Rat behavior at the rewarded end of the track involved horizontal sniffing and searching behavior with rapid chewing of the food pellets. Note that rats did not have to stop their searching behavior to chew, as the pellets were small enough to be held entirely in the mouth.

\section{Theta Power}

Observation of the EEG profile for a single given trip demonstrated that there existed a drop in theta power prior to the end of the locomotion behavior when approaching a rewarded location. This can be seen visually in EEG data when compared with speed. Figure 6 presents individual examples. Vertical lines in each EEG segment denote the times of maximal speed, the midpoint of deceleration and the minimal speed for that particular trial (left to right) as determined by fitting a curve to the speed data. For vectors of travel to the unrewarded end of the arm, the drop-off in theta power was far less severe.

Theta power and velocity data from all five rats were averaged together, timelocked to the ending of each trip, as determined by the algorithm that monitored position and speed. Figure 7 shows averaged speed and theta power for rewarded and unrewarded ends of the track for these five rats. Note the higher peak speed for ment point of both types of lever press are indicated with a stippled line. Error bars are for unweighted averages with one data point per time interval per rat $(n=6)$.

rewarded trials, but that deceleration occurs at a similar rate for the last $400 \mathrm{~ms}$ of both the rewarded and unrewarded trips, during the period when difference in theta power is large.

When theta power was compared to speed in segments of data that were timelocked to the end of a trip, a diminution of hippocampal theta power was much greater for trips to the rewarded end than the unrewarded end. The two theta power profiles shown in Figure 7 were found to be significantly different by a two-factor ANOVA with repetition $(\mathrm{F}(1,272)=298.64, P<0.00001)$. The interaction was also significant $(\mathrm{F}(33,272)=4.35, P<0.00001)$.

The difference in theta power between rewarded and unrewarded runs is significant at $240 \mathrm{~ms}(\mathrm{~T}(4)=-3.197, P<0.05$, two tailed) prior to the end of the run. At this time, theta power during rewarded trials continues to drop and reaches a minimum of $58 \%$ at about the same time that velocity reaches a minimum. In contrast, theta power drops to only $84 \%$ of maximum as the rat approaches the unrewarded end of the track. This period during which theta power is diverging between the two trial types is precisely when the velocity profiles are at their most similar, as shown in Figure 7B.

These data are normalized as described within the methods to be able to compare theta between rats with differing degrees of theta. A comparison of the averaged raw (unnormalized) theta power scores for the first time point in the analysis (a time near the maximal peak of the speed) indicated no significant difference between rewarded and unrewarded trips, i.e., $\mathrm{T}(4)=-0.06$, NS. 
A.

\section{EEG}

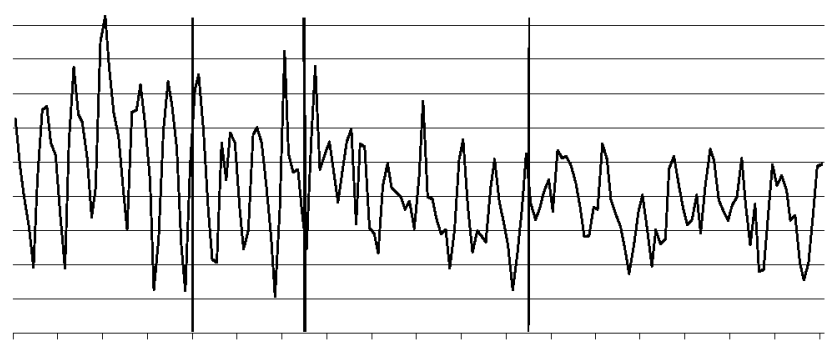

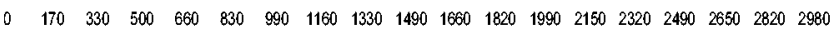

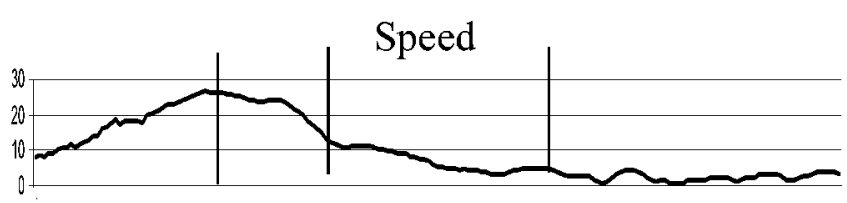

B.

EEG

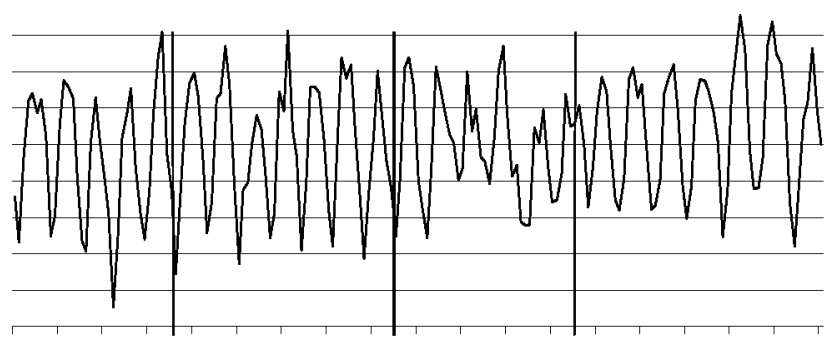

$0 \quad 170 \quad 330 \quad 500 \quad 660 \quad 830 \quad 990116013301490166018201990215023202490265028202980$

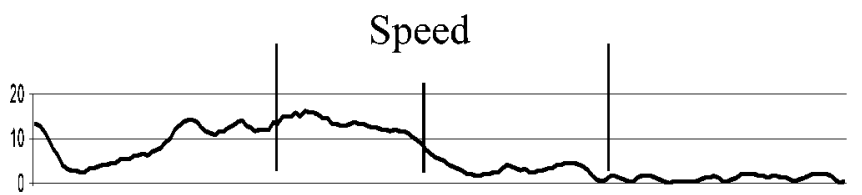

\section{Time relative to end of Run ( $\mathrm{msec}$ )}

FIGURE 6. Raw data showing theta recorded in a single rat at the rewarded end of the running task (A) vs the unrewarded end (B). Note that despite a similar drop in speed the two cases show a dramatic difference in amount of theta. Running speed is represented in inches/ second.

This indicates that the difference in the time course and magnitude of changes in theta power was not due to differences in initial theta power causing floor or ceiling effects. Rats had statistically similar theta power at top speed in both conditions even though speed was slightly higher for runs toward the rewarded end.

\section{DISCUSSION}

These experiments examined the time course of hippocampal theta power as it occurred during bar-pressing and running behaviors. For rewarded lever presses and also for approach to the rewarded end of the linear track these behaviors are associated with rapid reductions in theta power that begin several hundred milliseconds prior to completion of the motor activity. In the case of the conditional discrimination task, variations in theta power were also found between different learning epochs (chance vs well-learned behavior) for a bar press behavior that initiated a trial.

\section{Comparison With Existing Data}

Theta has often been reported to accompany voluntary movements, and there has been some debate about whether bar pressing falls into this category. Paxinos and Bindra (1970), Vanderwolf (1969), and Whishaw and Vanderwolf (1973) report a general correlation between motor activity and theta. Results in Dalton and Black (1968), Vanderwolf (1971), Whishaw et al. (1972), Black and Young (1972), and Buzsaki et al. (1985) specifically examine and report the observation of theta rhythm during bar presses. Two studies explicitly claim otherwise: both Feder and Ranck (1973) and Coleman and Lindsley (1975) report a very notable lack of theta oscillations during bar presses that have been performed many times, although the latter study was performed on
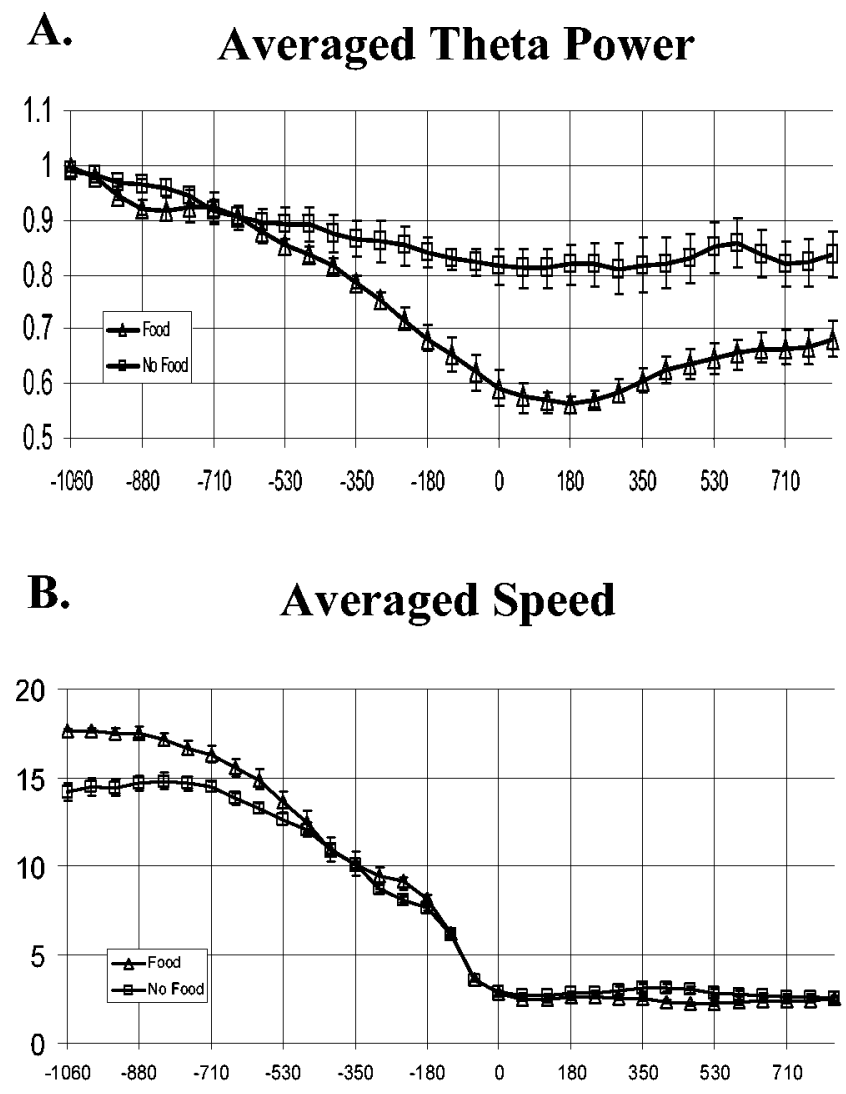

Time relative to end of Run (msec)

FIGURE 7. Averaged theta power at 60-ms intervals from all five rats for both rewarded (triangles "Food") and unrewarded (squares "No Food") runs (A), along with concurrent speed data from the same rats $(B)$. Note the $42 \%$ decline in theta power for the rewarded end of the track compared with the $18 \%$ decline for the unrewarded end. Error bars represent unweighted averages with one data point per time interval per rat $(n=5)$. Vertical axis units are in inches/second. 
cats. Both cite automation of the response as causing this drop in theta power.

The data in the present report address this debate by examining bar presses in two distinct behavioral contexts, finding theta present in the one, but absent in the other. While theta power did decrease over time due to habituation to the environment, theta still accompanied center lever presses even after 10,000 training trials. Further, in progressing from epoch 3 to epoch 5, theta power began to increase prior to center lever presses, which argues strongly against a habituation account. Simultaneously, lateral lever presses were consistently accompanied by a dramatic lack of theta activity throughout the entire course of the experiment. In the analysis described in this report, effort was taken to look for differences in theta power that might be attributed to differences in the specific motor activity used in performing them. In neither the case of invalid lever presses nor side biases was such a difference found. The strongest remaining possibility is that the difference in behavioral context was the reason for the difference in theta power profiles. The center lever press was used to initiate a trial, while the lateral lever was used to make a choice, thereby terminating a trial and leading to expectation of reward.

The properties of theta rhythm offset during running in the second experiment in this paper supports the results of the first experiment and provides further clarification of the relative time course of the cessation of theta activity and type 1 motor activity. It is well known that theta will accompany active running in the rat for hours on end (Whishaw and Vanderwolf, 1973). It has also been shown that there is a positive correlation between theta amplitude, frequency and running speed (Rivas et al., 1996). Less is known about the cessation of theta, a paradigm not well studied. Whishaw (1972) and Vanderwolf (1971) report that the hippocampal EEG exhibits small-amplitude irregular activity (SIA) during a rapid cessation of motor activity. Results describing a cessation of theta rhythm similar to that shown in the present study were described by Gray and Ball (1970), but not with the temporal precision required to provide a quantitative description of the time course of these changes. Kimsey et al. (1974) also performed a runway experiment, crossing expectation of reward or nonreward at the end of the alley with actual occurrence of reward. Their results indicated that the actual behavior at the end of the arm (consumption of reward) was the primary determinant of theta activity (measured by frequency, not power), as opposed to expectation of reward. However, their analysis did not focus on the time period immediately prior to arrival at the goal cup.

The data presented in this study could be seen as a contradiction of the standard view of hippocampal theta, in that it demonstrates a strong distinction between theta activity and motor activity. Theta drops sharply during deceleration in the approach to the rewarded end but not the unrewarded end. However, a clearer interpretation that fits in well with the sensorimotor integration hypothesis is that theta power changes precede motor activity by several hundred milliseconds. This has been shown for the initiation of theta and type 1 motor behavior (Vanderwolf, 1969), and in this report is shown for the termination of type 1 motor behavior.

\section{Theoretical Implications}

This discussion will make the following points. First, theta power seems to be more indicative of the future behavioral state than the current one, a finding that gives theta more of a role in the planning than the maintenance of motor activity. Second, the manner in which theta power profiles change during center lever press behavior is well described by the sensorimotor integration hypothesis of Bland and Oddie (2001).

The point made most strongly by these data is that theta power seems to correlate with the behavioral state several hundred milliseconds in the future. While this had been described before for the transition from a non-theta to a theta state (Vanderwolf, 1969), these data make the same point for transitions in the opposite direction. It is argued here that the difference in theta power during center and lateral lever presses is a result of the activity following the lever press. In the case of the center lever press, continuing type 1 motor behavior was necessary to complete a trial, and therefore theta levels remained high during this motor activity. In contrast, the lateral lever press was followed by a period of waiting for, and possibly consuming, a food reward. Therefore, theta activity dropped in anticipation of this change in behavioral state.

The results of the running experiment make the same point. At the unrewarded end of the track, the rats cease walking but continued to engage in exploratory sniffing behavior. Conversely, rats engaged in consummatory activity at the rewarded end of the track, an activity known to be accompanied by large-amplitude irregular activity (LIA, i.e., a lack of theta) (Green and Arduini, 1954; Vanderwolf, 1969). Theta activity in both cases reflected the behavioral state (theta or non-theta) several hundred milliseconds prior to the actual behavior itself. In both experiments, the ongoing motor activity (bar pressing and running) during periods of theta and non-theta EEG was identical. The most obvious difference between the two is the future behavioral state of the animal: this future state is continued type 1 theta behaviors in the case of center lever pressing and approach to the unrewarded end of the track; and the future state is consummatory, non-theta activity in the case of pressing the lateral lever and arriving at the rewarded end of the track. The effect of behavioral context on the degree of theta expression during a given activity is also discussed in van Lier et al. (2003).

This perspective casts the role of theta mechanisms in motor activity as one of planning and initiation, very similar to the description of the role of type 2 , as described in Bland and Oddie (2001). If theta is essential for engaging a type 1 motor activity, it is not necessary during the final $200-300 \mathrm{~ms}$ of performance.

This idea fits well with the sensorimotor integration hypothesis of Bland and Oddie (2001) and these data extend its explanatory power to cover the conclusion of a sequence of motor activity. The sensorimotor integration hypothesis states that type 2 theta activity is generated by cholinergic mechanisms originating in the medial septum and serves as a means of priming the motor system for activity, a "readiness signal." If motor activity does ensue, type 1 theta (atropine resistant) mechanisms are engaged in conjunction with the motor activity. The description of the sensorimotor integration theory as given in Bland and Oddie (2001) focuses primar- 
ily on the initiation of type 1 voluntary motor activity. The data presented in the present report make a strong case that just as theta activity precedes the initiation of motor activity, a lack of theta power precedes non theta activities.

The change of the center lever theta profile between well-learned (epoch 3) and chance behavior (epochs 1 and 5) support the sensorimotor integration theory as well. Theta power was shown to increase just prior to the cue light during those trials in which that cue light appears to be partially controlling behavior.

A report by Bland et al. (1999) is also relevant here, in describing the mechanics of transitions between the theta and non theta state in both directions by analyzing the rhythmic activity of single cells in the hippocampus. In that article, the point is made that non-theta to theta transitions appear to require 500 $\mathrm{ms}$ as theta-ON cells are gradually recruited. In contrast, theta to non-theta transitions involve the very rapid disinhibition of theta-OFF cells, which abruptly shuts down theta activity. This asymmetry in the initiation and termination of theta is reflected in these data by gradual increases in theta activity observed prior to center lever presses and dramatic and rapid reductions in theta activity observed prior to lateral lever presses and arrival at the rewarded end of the alley.

There is a small decline in theta power at the moment of the center lever bar press that is unique to epoch 3. Informal behavioral observations of the rats are useful in explanation of this difference. During periods of well learned behavior, rats tended to press the center lever and then remain reared and very still while waiting for the cue lights (400-700-ms random delay following the center lever press). If one considers the center lever press to be the end of a sequence of motor activity during this epoch, as described for the lateral lever in the previous section, one would expect to see a drop in theta power during this epoch as well. As the expected time of the cue light nears, theta power begins to increase in preparation for committing a response, integrating the sensory and motor components of the task in the manner described by Bland and Oddie (2001). In effect, both behavior and theta power suggest that during well learned behavior, the rat effectively separated the trial into two discrete stages, first initiating a trial with the center lever task, and then pausing to wait for the cue lights in order to complete the trial.

During epochs 1 and 5, when rats were not behaviorally discriminating between the lights, behavior tended to involve a continuous series of motor action from center lever to lateral lever. When the rat did pause in the reared position on the center lever during these chance behavior epochs, it did not appear to be observing the lights (based on head orientation that differed from what was observed during epoch 3 ). We suggest the possibility that during these epochs, rats were not taking sensory information into account. Instead they may have been trying to encode the bar presses as a form of response memory (Numan and Klis, 1992). Accordingly, the entire trial, from center lever to lateral lever was a single motor sequence and therefore failed to exhibit the theta power reduction during the center lever press observed in epoch 3 .

\section{Consummatory Behavior and Theta: Implications for Attention}

A subtle aspect of these data concerns EEG recorded during consummatory behavior. While receiving reward at the food end of the track, the act of eating was extremely short in duration and was accompanied by exploratory sniffing to locate food pellets prior to, during, and following the actual chewing. Certainly some type 1 motor programs were strongly engaged by this behavior. Despite this qualitative similarity in behavior at both rewarded and unrewarded ends of the track, there was a strong reduction in theta prior to and during the acquisition of the two food pellets. This finding indicates the possibility that theta activity is incompatible or at least strongly anticorrelated with motor programs responsible for consummatory activities. There may be a mechanism for shutting down the production of both type 1 and type 2 theta during particular non-theta activities, even if there is some degree of ongoing type 1 motor activity. However, this viewpoint is contradicted by reports in Vanderwolf (1969), which describe theta activity during consumption of large food pellets, presumably the result of forelimb movements activating type 1 theta generation mechanisms.

The control of behavior is a complex process and may be governed by multiple parallel systems. For example, Sinnamon (1993) describes three different locomotor systems - exploratory, appetitive, and defensive- each with their own diencephalic mechanisms. If true, this indicates that the primary motive behind any given locomotor behavior will be a critical factor in the concurrent function of higher-level brain systems. One would expect the functional state of the telencephalon to vary considerably between even similar "voluntary" movements associated with exploration, consummatory activity, and defensive behaviors.

For example, it may be the case that a drop in theta power during consumption is indicative of a state of hypervigilance to any possible sensory input for purposes of detecting nearby predators. This idea of non-theta activity representing a state of hippocampal sensitivity to generalized sensory inputs is based on and strongly supported by hippocampal single-cell data from Vinogradova (1995). This framework also broadly fits the theoretical observations of Sainsbury (1998) and Grastyan et al. (1959) that hippocampal theta activity may represent a state of inhibition, not arousal. Behavioral evidence of such vigilance is directly supported by observations of hyperreactivity to sensory stimuli during atropine infusions of the medial septum (Lawson and Bland, 1993), which cause reductions in hippocampal theta activity during immobility. This nonspecific attention could be optimized for use in detecting the approach of predators during consumption of food or water in species such as the rat. Food-rich areas in an actual environment may be more likely to be frequented by predators, especially if the animal is forced to eat outside of its nest, as was the case in this experiment.

Theta, on the other hand, may indicate a primary activation of an exploratory locomotor system, focusing the hippocampal system to specific types of sensory stimuli, with the intent of learning information about the environment. This selectivity might be accomplished by aligning salient sensory stimuli to a specific phase of 
theta using timing mechanisms external to the hippocampus (Vinogradova, 1995; Givens, 1996; Hasselmo et al., 2000, 2002).

This framework essentially posits two different kinds of attention. There exists a hypervigilant, nonspecific attention that is incompatible with cholinergic hippocampal function and is active in situations during which any possible sensory stimulus needs to be detected. The other form of attention would be a theta-dependent, focused attention that is optimized for learning about specific sensory inputs and is engaged during exploratory behavior, operant conditioning, and any situation in which a salient sensory stimulus is ongoing (Sainsbury, 1998).

\section{Final Discussion}

Combined neuronal and EEG recording demonstrates that there are important differences in the way that theta activity in the hippocampus is initiated and shut down (Bland et al., 1999). Many reports exist concerning the way that theta correlates with the initiation of motor activity (for review, see Bland and Oddie, 2001). The incidence of this type 2 theta prior to concurrent type 1 theta and type 1 motor activity is one of the cornerstones on which the sensorimotor integration hypothesis is founded. However, comparatively few data exist about how theta power relates to the cessation of motor activity. This report clearly makes the point that theta cessation precedes the cessation of motor activity in two different paradigms: operant bar pressing and running behavior.

In conjunction with reports indicating the increase of theta frequency prior to initiation of type 1 motor activity (Vanderwolf, 1969), the data presented in the present report indicate that changes in theta power always seems to precede changes in type 1 motor activity by several hundred milliseconds, whether that change be initiation or termination. If correct, this means that the disappearance of hippocampal theta power during bar pressing (Feder and Ranck, 1973) is a function of the relative position of the bar press response in the motor sequence. If the bar press represents the final part of such a sequence and is followed by an LIA (nontheta) activity (in the case of this report, consummatory behavior), theta power will decrease abruptly $200-400 \mathrm{~ms}$ in advance of the completion of the motor activity. The second experiment in this study confirms that the same principle appears to apply to theta accompanying running behavior as well.

\section{REFERENCES}

Berry SD, Seager MA. 2001. Hippocampal theta oscillations and classical conditioning. Neurobiol Learn Mem 76:298-313.

Black AH, Young GA. 1972. Electrical activity of the hippocampus and cortex in dogs operantly trained to move and to hold still. J Comp Physiol Pyschol 79:128-141.

Bland BH, Oddie SD. 2001. Theta band oscillation and synchrony in the hippocampal formation and associated structures: the case for its role in sensorimotor integration. Behav Brain Res 127:119-136.

Bland BH, Oddie SD, Colom LV. 1999. Mechanisms of neural synchrony in the septohippocampal pathways underlying hippocampal theta generation. J Neurosci 19:3223-3237.
Buzsaki G, Rappelsberger P, Kellenyi L. 1985. Depth profiles of hippocampal rhythmic slow activity ("theta rhythm") depend on behaviour. Electroencephalogr Clin Neurophysiol 61:77-88.

Caplan JB, Madsen JR, Raghavachari S, Kahana MJ. 2001. Distinct patterns of brain oscillations underlie two basic parameters of human maze learning. J Neurophysiol 86:368-380.

Clody DE, Carlton PL. 1969. Behavioral effects of lesions of the medial septum of rats. J Comp Physiol Psychol 67:344-351.

Coleman JR, Lindsley DB. 1975. Hippocampal electrical correlates of free behavior and behavior induced by stimulation of two hypothalamichippocampal systems in the cat. Exp Neurol 49:506-528.

Dalton A, Black AH. 1968. Hippocampal electrical activity during the operant conditioning of movement and refraining from movement. Commun Behav Biol 196(Pt A):267-273.

Feder R, Ranck JB Jr. 1973. Studies on single neurons in dorsal hippocampal formation and septum in unrestrained rats. II. Hippocampal slow waves and theta cell firing during bar pressing and other behaviors. Exp Neurol 41:532-555.

Givens B. 1996. Stimulus-evoked resetting of the dentate theta rhythm: relation to working memory. NeuroReport 8:159-163.

Grastyan E, Lissak K, Madarasz I, Donhoffer H. 1959. Hippocampal electrical activity during the development of conditioned reflexes. Electroencephalogr Clin Neurophysiol 11:409-430.

Gray JA, Ball GG. 1970. Frequency-specific relation between hippocampal theta rhythm, behavior, and amobarbital action. Science 168:1246-1248.

Green JD, Arduini AA. 1954. Hippocampal electrical activity in arousal. J Neurophysiol 75:533-557.

Hasselmo ME, Bodelon C, Wyble BP. 2002. A proposed function for hippocampal theta rhythm: separate phases of encoding and retrieval enhance reversal of prior learning. Neural Comput 14:793-817.

Hasselmo ME, Fransen E, Dickson C, Alonso AA. 2000. Computational modeling of entorhinal cortex. Ann NY Acad Sci 911:418-46.

Irmis F, Radil-Weiss T, Lat J, Krekule I. 1970. Inter-individual differences in hippocampal theta activity during habituation. Electroencephalogr Clin Neurophysiol 28:24-31.

Kahana MJ, Sekuler R, Caplan JB, Kirschen M, Madsen JR. 1999. Human theta oscillations exhibit task dependence during virtual maze navigation. Nature 399:781-784.

Kimsey RA, Dyer RS, Petri HL. 1974. Relationship between hippocampal EEG, novelty, and frustration in the rat. Behav Biol 11:561-568.

Kramis R, Vanderwolf CH, Bland BH. 1975. Two types of hippocampal rhythmical slow activity in both the rabbit and the rat: relations to behavior and effects of atropine, diethyl ether, urethane, and pentobarbital. Exp Neurol 49(1 Pt 1):58-85.

Lawson VH, Bland BH. 1993. The role of the septohippocampal pathway in the regulation of hippocampal field activity and behavior: analysis by the intraseptal microinfusion of carbachol, atropine, and procaine. Exp Neurol 120:132-144.

Monmaur P, Breton P. 1991. Elicitation of hippocampal theta by intraseptal carbachol injection in freely moving rats. Brain Res 544:150 155.

Numan R, Klis D. 1992. Effects of medial septal lesions on an operant delayed go/no-go discrimination in rats. Brain Res Bull 29:643-650.

Paxinos G, Bindra D. 1970. Rewarding intracranial stimulation, movement and the hippocampal theta rhythm. Physiol Behav 5:227-231.

Rivas J, Gaztelu JM, Garcia-Austt E. 1996. Changes in hippocampal cell discharge patterns and theta rhythm spectral properties as a function of walking velocity in the guinea pig. Exp Brain Res 108:113-118.

Sainsbury RS. 1970. Hippocampal activity during natural behavior in the guinea pig. Physiol Behav 5:317-324.

Sainsbury RS. 1998. Hippocampal theta: a sensory-inhibition theory of function. Neurosci Biobehav Rev 22:237-241.

Sainsbury RS, Harris JL, Rowland GL. 1987a. Sensitization and hippocampal type 2 theta in the rat. Physiol Behav 41:489-493.

Sainsbury RS, Heynen A, Montoya CP. 1987b. Behavioral correlates of hippocampal type 2 theta in the rat. Physiol Behav 39:513-519. 
Sarnthein J, Petsche H, Rappelsberger P, Shaw GL, von Stein A. 1998. Synchronization between prefrontal and posterior association cortex during human working memory. Proc Natl Acad Sci USA 95:70927096.

Sinnamon HM. 1993. Preoptic and hypothalamic neurons and the initiation of locomotion in the anesthetized rat. Prog Neurobiol 41:323344.

van Lier H, Coenen AM, Drinkenburg WH. 2003. Behavioral transitions modulate hippocampal electroencephalogram correlates of open field behavior in the rat: support for a sensorimotor function of hippocampal rhythmical synchronous activity. J Neurosci 23:2459-2465.

Vanderwolf CH. 1969. Hippocampal electrical activity and voluntary movement in the rat. Electroencephalogr Clin Neurophysiol 26:407418.

Vanderwolf CH. 1971. Limbic-diencephalic mechanisms of voluntary movement. Psychol Rev 78:83-113.

Vinogradova OS. 1995. Expression, control, and probable functional significance of the neuronal theta-rhythm. Prog Neurobiol 45:523-583.
Walter VJ, Walter WG. 1953. The central effects of rhythmic sensory stimulation. Electroencephalogr Clin Neurophysiol 1:57-86.

Whishaw IQ. 1972. Hippocampal electroencephalographic activity in the Mongolian gerbil during natural behaviours and wheel running and in the rat during wheel running and conditioned immobility. Can J Psychol 26:219-239.

Whishaw IQ, Vanderwolf CH. 1973. Hippocampal EEG and behavior: changes in amplitude and frequency of RSA (theta rhythm) associated with spontaneous and learned movement patterns in rats and cats. Behav Biol 8:461-484.

Whishaw IQ, Bland BH, Vanderwolf CH. 1972. Hippocampal activity, behavior, self-stimulation, and heart rate during electrical stimulation of the lateral hypothalamus. J Comp Physiol Psychol 79:115-127.

Winson J. 1974. Patterns of hippocampal theta rhythm in the freely moving rat. Electroencephalogr Clin Neurophysiol 36:291-301.

Wyble BP, Linster C, Hasselmo ME. 2000. Size of CA1-evoked synaptic potentials is related to theta rhythm phase in rat hippocampus. J Neurophysiol 83:2138-2144. 